\title{
A cellulosic paper-based sensor for detection of starch contamination in milk
}

\author{
ARUN KUMAR GOVINDARAJALU ${ }^{1}$, MUTHAMILSELVI PONNUCHAMY ${ }^{1}$, \\ BALASUBRAMANIAN SIVASAMY1, M VENKATESH PRABHU ${ }^{2}$ and ASHISH KAPOOR ${ }^{1, *(D)}$ \\ ${ }^{1}$ Department of Chemical Engineering, SRM Institute of Science and Technology, Kattankulathur 603 203, India \\ ${ }^{2}$ Department of Biotechnology, SRM Institute of Science and Technology, Kattankulathur 603 203, India \\ *Author for correspondence (ashishko@srmist.edu.in)
}

MS received 20 October 2018; accepted 26 March 2019

\begin{abstract}
Chemical and biomolecular analysis form an integral part of food contamination testing and healthcare diagnostics. The majority of the currently established detection methods are expensive, sophisticated and time consuming. Microfluidic-based lab-on-a-chip systems offer a promising alternative in this regard by enabling the development of simpler and relatively cost-effective platforms for analytical applications. In this work, we report the fabrication of a portable sensor platform using selective wax impregnation of cellulosic paper. The application of the fabricated paper-based lab-on-achip device is demonstrated for the qualitative and quantitative detection of starch contamination in milk using colorimetry. Integration of a detection procedure with smartphone imaging allows for on-the-spot data collection, thus overcoming the challenges of sample storage, handling and transportation to lab facilities. This approach offers a versatile method for diagnostic and sensing applications in resource-limited areas, especially in developing and underdeveloped countries.
\end{abstract}

Keywords. Paper-based sensor; microfluidics; starch contamination; milk; detection.

\section{Introduction}

Affordable and accessible analytical techniques for the detection of contaminants in food and environmental resources are essential for health and well-being of every society. Food quality testing, healthcare diagnostics and environmental monitoring technologies have progressed at a rapid rate over the recent years. However, the majority of state-of-theart analytical and diagnostic techniques still remain elusive for a large section of world population. This is due to several reasons, including requirement of sophisticated equipment, expensive reagents, difficulty in sample handling and need for trained personnel [1]. As a consequence, various contaminants and pathogens in our food and environment remain undetected. The lack of timely detection of contaminants results in undesirable aggravation of health problems in individuals, infectious spreading and mortality [2].

Milk is one of the most essential foods of human beings. Being a source of all vital nutrients, it is ubiquitously consumed by children and adults in various forms. Contamination of milk has been an historical issue and is a major health concern. National survey on milk adulteration conducted by the Food Safety and Standards Authority of India (FSSAI) in 2011 revealed a plethora of contaminants in milk throughout India. One of such contaminants is starch that is usually added to increase solid non-fat content of milk. Excessive amounts of starch in milk remain undigested and are reported to cause diarrhea [3]. Furthermore, intensified levels of starch accumulation in body may prove to be fatal for patients suffering from diabetes. Although, milk quality testing methods have been reported [4], most of them are qualitative in nature and need careful handling of reagents that limit their widespread usage by general populace. The motivation to develop a kit for the detection of starch adulteration in milk is to overcome some of the limitations of the conventional methodology and make the assay portable, affordable and more easily accessible for the larger section of society. The conventional approach requires collection and transportation of milk samples to laboratory followed by an analytical reaction in the test tube. A few primary limitations of the prevalent approach are listed here. The time delay involved between collection of milk from the source and its analysis in laboratory can affect sample quality and give false results. The test tube method requires dealing with considerable amounts of reagents and samples. The nature of reagents requires careful handling by trained professionals. These aspects also contribute to the increased costs associated with sample collection, storage, transport and analysis. The gravity of the situation calls for an increasing need for rapid, sensitive and affordable detection methods [5].

Recently, microfluidic-based lab-on-a-chip devices have emerged as a potential solution to the development of portable and economical diagnostic and detection platforms [6-8]. The lab-on-a-chip approach refers to miniaturization of one or more functions conventionally carried out in laboratories [9]. Typical functions include, though are not limited to, 
sample preparation, purification, extraction, reaction, detection and analysis. Several lab-on-a-chip devices or platforms have been reported for applications in chemical and biological sciences, drugs and pharmaceuticals, food quality testing and environmental sensing [10-13]. Such devices allow drastic amounts of reduction in reagent volumes, higher surface area to volume ratios, easier operations and reduced cost of fabrication as well as operation. Depending on applications, the devices have been fabricated using glass, acrylic, polydimethylsiloxane, hydrogel, etc. [7,14]. Recent research work has showcased emerging capabilities in the fabrication of lab-on-a-chip devices with commonly available substrates, including cellulosic paper [15-17]. In a broad perspective, the development of lab-on-a-chip platforms requires the selection of suitable materials as substrates, fabrication of a platform, surface functionalization, testing the feasibility of a process on a miniaturized scale, data collection and analysis.

In this work, our objective is to develop a portable analytical lab-on-a-chip platform on a cellulosic paper substrate using a wax patterning approach and demonstrate its application for the on-the-spot detection of starch contamination in milk. The proposed procedure is examined and established to be apt for quantifying the results apart from qualitative yes/no detection outcome. The approach offers a viable and versatile method for contamination detection applications in resource-limited regions.

\section{Materials and methods}

\subsection{Materials and equipment}

The design of a detection platform comprises hydrophilic circular regions confined by hydrophobic boundaries. Standard filter paper (Whatman qualitative \#1) was used as a substrate for making the devices. Solid paraffin wax was sourced from a local commercial shop in Chennai, India. Wax was selected for making hydrophobic barriers on a paper substrate due to its suitable characteristics. It readily melts on exposure to heat and permeates through the porous matrix of the substrate. Upon cooling, it solidifies and occupies the pores thus enabling the formation of hydrophobic barriers. Milk was sourced from a local commercial dairy store. Potassium iodide (KI) and iodine were used as chemical reagents for the colorimetric detection of starch in milk. KI contains iodide ions that combine with iodine molecules to form tri-iodide ions that are soluble and are responsible for interaction with starch resulting in colorimetric signal generation. All chemicals used in the experiments were of analytical grade and were purchased from the Southern India Scientific Corporation, Chennai, India. Double distilled water was used for all dilutions. Commercially available ink purchased from a local stationery shop (Chennai, India) was used for the purpose of confirmation of the hydrophilic detection zone. A smartphone (Samsung Galaxy s7e, model number SM-G935FD, Samsung India) was used for image acquisition during the experiments.

\subsection{Fabrication}

The fabrication of a cellulosic paper-based analytical lab-ona-chip platform was performed using the principles of wax permeation as reported in the literature [18,19]. Figure 1 shows the steps involved in fabrication. Briefly, the paper substrate was marked with the design of the detection region, here circular in shape. The design was printed or drawn manually using a marker. Wax was then gently rubbed outside the boundaries of the detection region. The substrate with wax coating was placed on a hot plate and temperature was set to $120^{\circ} \mathrm{C}$ for $5 \mathrm{~s}$. Heat treatment caused wax to melt and permeate across the thickness of paper. The substrate was allowed to cool down for $10 \mathrm{~min}$ and stored in a clean and dry environment for further experiments.

\subsection{Experimental}

Ink solution was used to validate the formation of hydrophilic detection regions surrounded by hydrophobic boundaries. A total of $200 \mu \mathrm{l}$ of ink was pipetted and gently placed in the centre of the detection region. It was allowed to diffuse and observations were visually and photographically recorded.

For milk contamination testing experiments, $1 \%$ iodine solution was prepared using KI and iodine. A total of $200 \mu \mathrm{l}$ of the solution of potassium tri-iodide was coated in the detection region and allowed to dry for $5 \mathrm{~min}$. The devices were stored or used for subsequent testing. Milk procured from the store was used as a blank sample. Another contaminated milk sample was synthetically prepared for testing purposes by dissolving a pre-measured amount of starch in milk. A total of $200 \mu \mathrm{l}$ of sample was pipetted and gently introduced at the centre of the detection region and colour changes were recorded. Miniscule amounts of reagents and samples were required for performing lab-on-a-chip experiments compared to a macroscale. For further quantitative analysis, images were captured using a smartphone and processed as described in the subsequent sub-section. In order to generate a calibration graph for various concentrations of starch in milk, a series of experiments were performed for starch concentration ranging between 0 and $10 \% \mathrm{~W} \mathrm{v}^{-1}$.

\subsection{Image processing and analysis}

The images of the experiments were acquired with a mobile phone camera without flash under good lighting conditions. The images were captured and processed inside laboratory. The light intensity around the working area was recorded before and after the experiments. The captured images were transferred to a personal computer, imported and analysed with the help of image processing software (Adobe Photoshop, USA). The size of each image was normalized and the working area was marked with the help of a circular marquee tool. The working area was isolated into a new layer so that it could be further processed. The average 


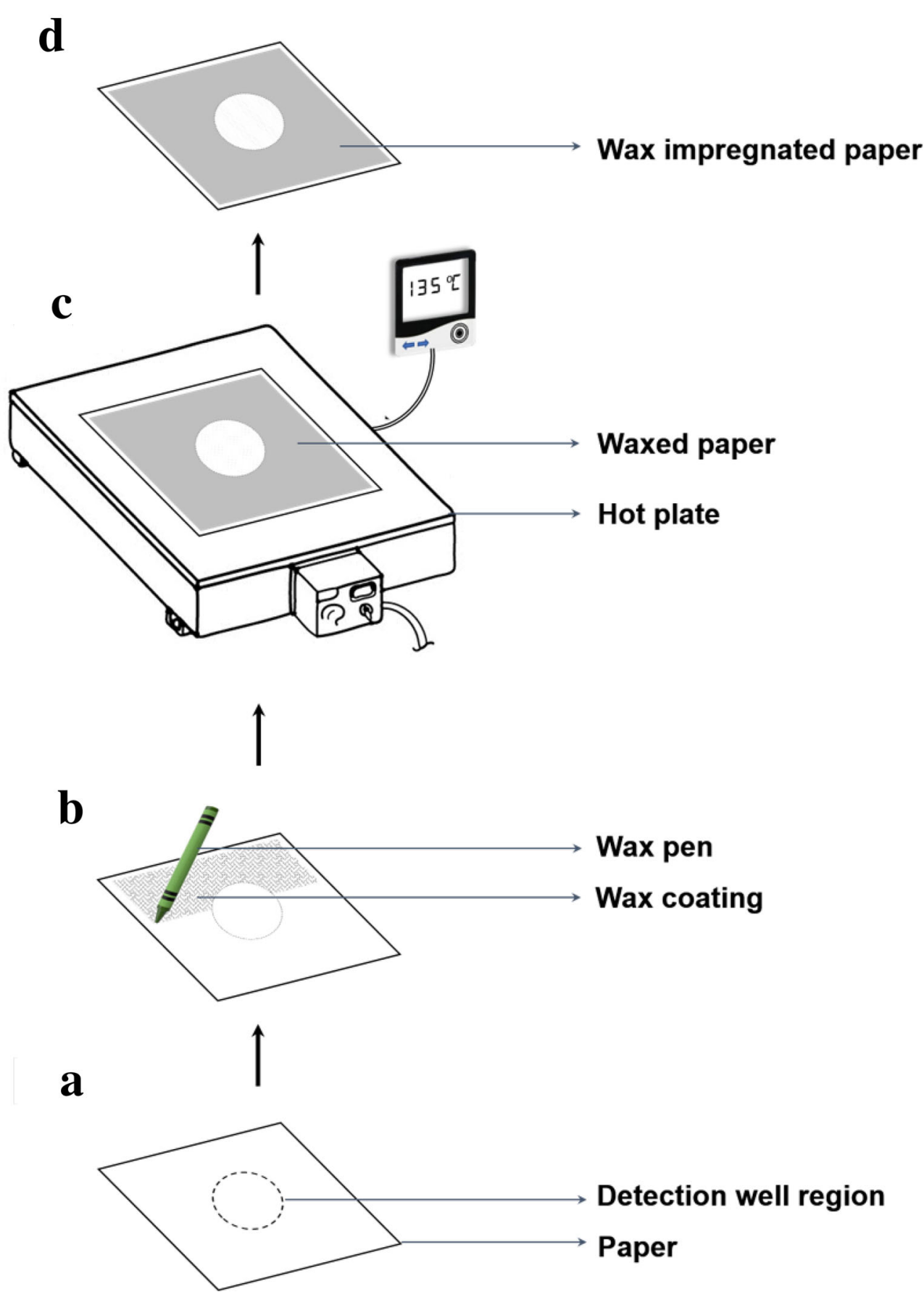

Figure 1. Steps involved in the fabrication of a paper-based sensor platform. (a) Marking of the detection region on paper, (b) wax coating around the region, (c) thermal treatment of wax on a hot plate and (d) final platform with the circular detection region surrounded by the wax impregnated region.

colour was obtained with the help of inbuilt tools and the standard red, green and blue (RGB) values were obtained with the help of a colour picker tool. The images were saved to a predefined location for future reference. The analysis was performed for all the experiments repeated in triplicates.

\section{Results and discussion}

\subsection{Validation of detection zone formation}

The formation of hydrophilic detection regions was confirmed using readily available ink solution. Ink solution diffuses from 
the centre of the region towards the periphery in the radial direction. The diffusion stops when the fluid faces the wax boundary, thus confirming the confinement on all sides of detection regions (figure 2).

\subsection{Colorimetric detection}

The presence of starch contamination in the milk sample was confirmed by a colour change in the detection regions.

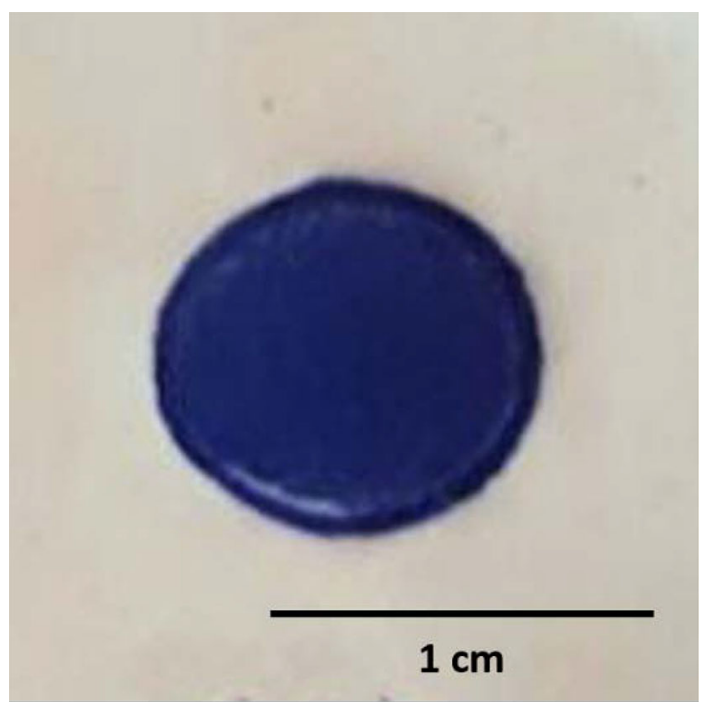

Figure 2. Demonstration of hydrophobic confinement using ink solution.

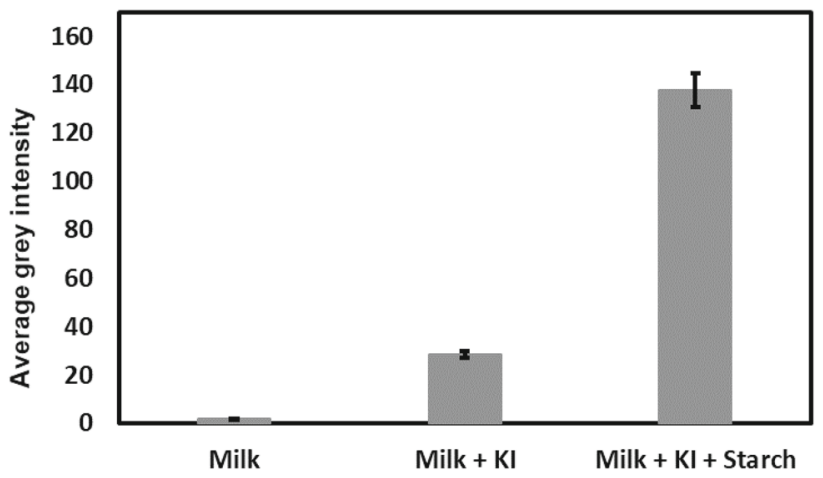

Figure 4. Quantification of colorimetric data for the detection of starch in milk.

This is a direct qualitative test for the presence of starch in the sample. Iodine by itself is sparingly soluble in water due to its non-polar nature. However, in the presence of KI, a linear tri-iodide ion complex is formed, which is soluble. This linear tri-iodide slips into the helical coil structure of starch giving dark bluish brown colour. A typical colorimetric response of addition of the milk sample on a paper-based analytical platform is shown in figure 3. The substrate in figure 3a corresponds to uncoated paper and those in figure $3 \mathrm{~b}$ and $\mathrm{c}$ correspond to paper coated with potassium tri-iodide solution. Addition of pure milk (figure $3 a$ and $b$ ) and starch contaminated milk (figure 3c) show evident colour transformations under different experimental conditions, thus

a
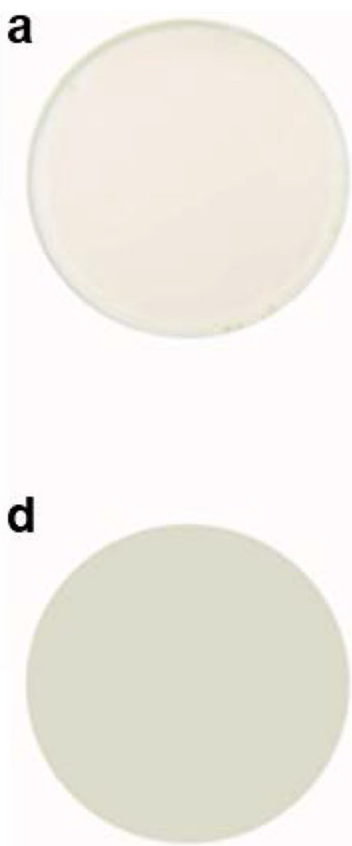

b
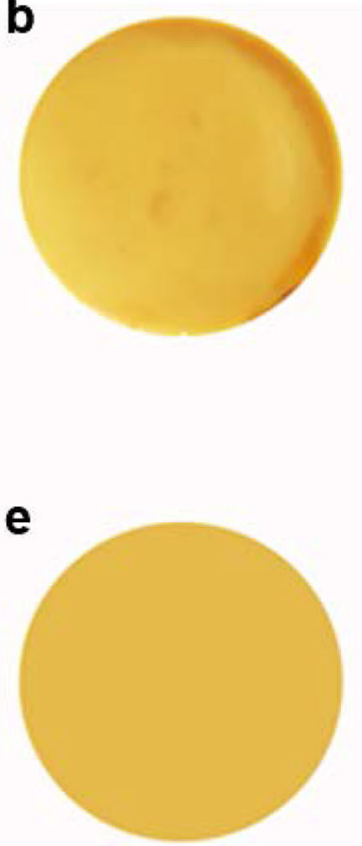
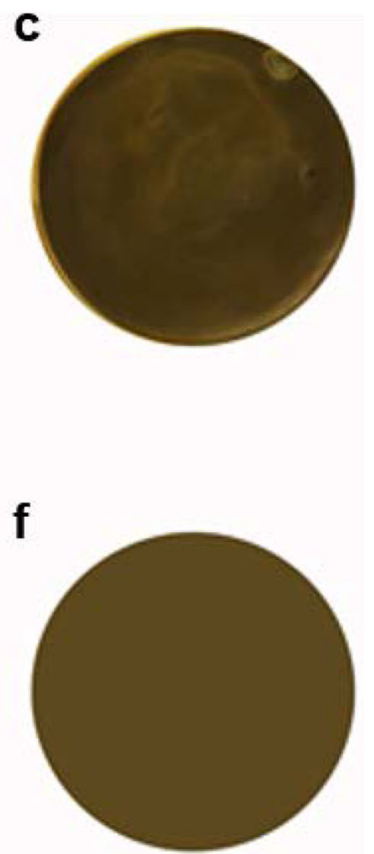

Figure 3. Colorimetric response of addition of milk samples for the detection region of paper-based analytical platforms for the cases: (a) pure milk on uncoated paper, (b) pure milk on paper coated with iodine+KI, (c) starch contaminated milk on paper coated with iodine+KI. Images (d), (e) and (f) correspond to averaged colour images for (a), (b) and (c), respectively. 
confirming the absence or presence of starch. A visual change in the colour signal is a qualitative way of starch detection. Furthermore, it can be quantified as described in the following sub-section.

\subsection{Quantitative analysis}

The processed images corresponding to the experiment described above are shown in figure $3 \mathrm{~d}-\mathrm{f}$. The grey scale colour intensity is obtained from the measured red, blue and green colour intensity data by using equation (1):

$$
\mathrm{GSI}=0.299 * \mathrm{R}+0.587 * \mathrm{G}+0.114 * \mathrm{~B},
$$

where GSI is the grey scale intensity and R, G and B denote the intensity of red, green and blue colour channels, respectively [20]. Pure milk is white in colour and serves as the baseline for estimating average grey intensity values. All subsequent average grey intensity values are reported with reference to
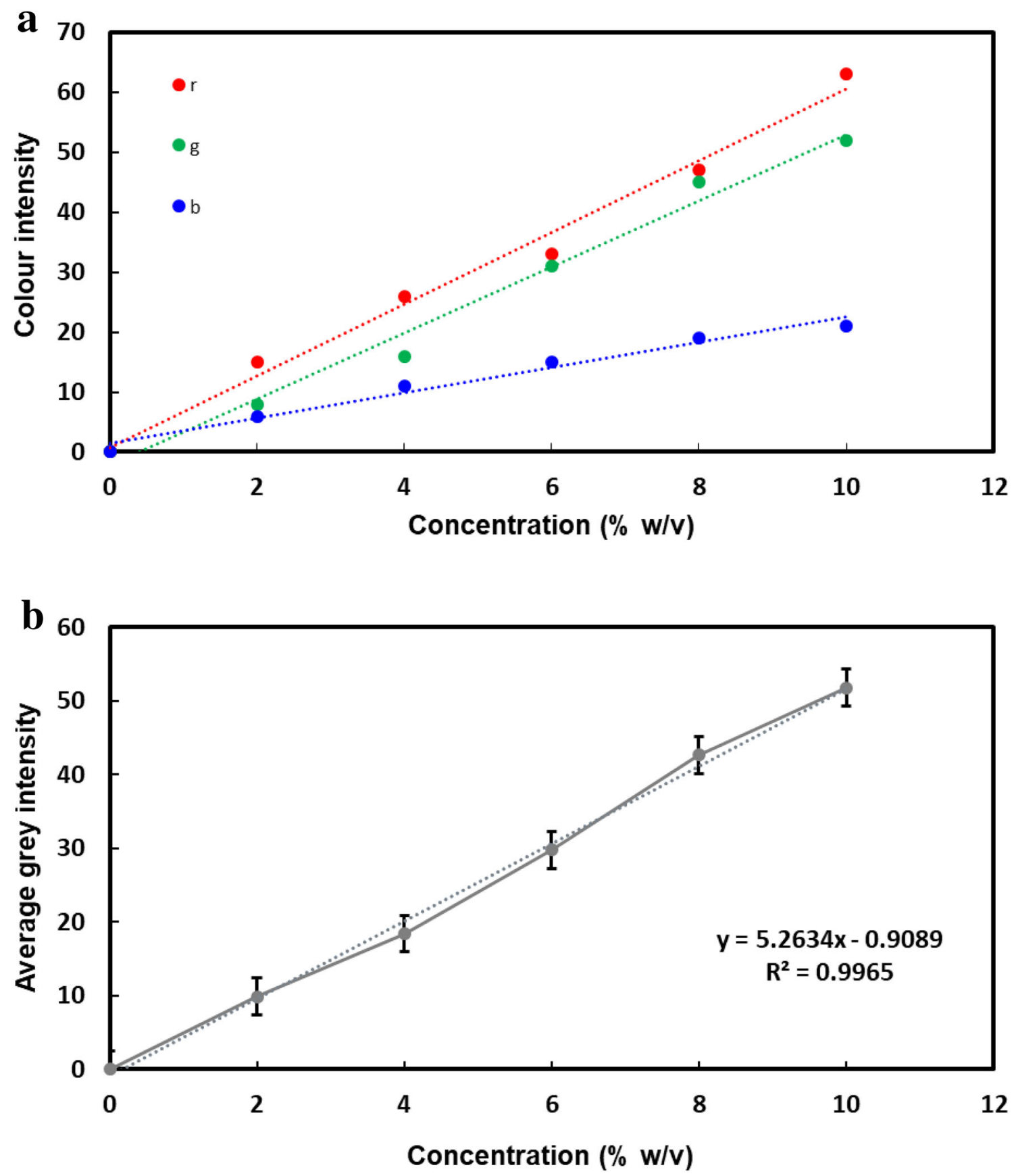

Figure 5. Calibration graph for: (a) colour intensity for red (r), green (g) and blue (b) colour channels and (b) average grey intensity for various concentrations of starch in milk. 
the baseline value. After adding iodine-KI solution, milk turns yellow and shows a slight increase in colour intensity, whereas, milk with starch shows a large increase in grey intensity because of bluish brown colour. These useful data can be used to estimate starch content. The average grey intensity values for the typical experiment described in figure 3 are shown in figure 4.

Similar analysis was performed for all images acquired during the calibration experiments. As it is evident from figure 5, colour intensity in the detection wells increased with successive increments in concentration. Both RGB intensity values and average grey intensity values are shown in figure 5a and b, respectively. The $y$-axis on the plots represents normalized colour intensity values. Standard deviations for multiple measurements are shown in the plots. Relative changes in intensity levels for RGB channels can be helpful in the selection of an appropriate channel for colorimetric-based detection [21]. The data shown here suggest that different channels show different variations for the same change in concentration levels. However, the grey scale is recommended to avoid subjectivities of different observers. The grey scale intensity data points are fitted and the linear equation $(y=5.2634 x-0.9089)$ is found to be the best fit $\left(R^{2}=0.9965\right)$. The calibration plot can be readily used to determine the starch concentration in unknown milk samples using the platform when imaged under standard conditions. The merits offered by integration of the paper-based sensor with smartphone imaging allow rapid data collection, analysis, interpretation and communication to the user, thus making the device a promising candidate for commercial acceptability.

Food contamination by natural or anthropogenic causes is a serious health concern. Starch, being one of the commonly reported contaminants in milk, must be monitored to avoid unwanted health implications [22]. An iodine-based test is an established method for the detection of starch [23]. However, it is usually performed in chemical, biological or food laboratory considering the reagent requirements and handling and safety concerns. The milk sample to be examined needs to be collected in field, followed by storage and transportation under controlled conditions in the lab. Any chemical transformation in the sample due to delay between sample collection and analysis can lead to false results. Such constraints are readily avoided with the detection test procedure described here on portable paper-based lab-on-a-chip platforms. The simplicity of the platform allows it to be easily fabricated and makes it apt for on-the-spot use without any necessity of special training.

\section{Conclusion}

This study demonstrates the development of a paper-based analytical platform for point-of-use applications. The device is made using inexpensive and commonly available raw materials, paper and wax, without the use of sophisticated equipment. It provides a portable and affordable alternative to conventional diagnostic and detection platforms. Image capturing using smartphones and processing allows us to obtain qualitative as well as quantitative results as per requirements. A proof-of-concept application is shown here by testing the feasibility of starch contamination detection in milk on the lab-on-a-chip platform. Similar strategies can be adopted for the detection of other chemical and biomolecular species.

\section{Acknowledgements}

The authors gratefully acknowledge SRM Institute of Science and Technology for the use of services and facilities throughout the research work. The authors also thank Dr Prabhakar for insightful discussions.

\section{References}

[1] Martinez A W, Phillips S T, Whitesides G M and Carrilho E 2010 Anal. Chem. 823

[2] Cuevas L E 2011 Indian J. Pediatr. 78449

[3] Azad T and Ahmed S 2016 Int. J. Food Contam. 322

[4] Singuluri H and Sukumaran M K 2014 J. Chromatogr. Sep. Tech. 5212

[5] Peeling R W, Smith P G and Bossuyt P M 2010 Nat. Rev. Microbiol. $4 \mathrm{~S} 2$

[6] Whitesides G M 2006 Nature 442368

[7] Mao X and Huang T J 2012 Lab Chip. 121412

[8] Gong M M and Sinton D 2017 Chem. Rev. 1178447

[9] Whitesides G 2014 Lab Chip. 143125

[10] Chiu D T, deMello A J, Carlo D D, Doyle P S, Hansen C, Maceiczyk R M et al 2017 Chem. 2201

[11] Dittrich P S and Manz A 2006 Nat. Rev. Drug Discov. 5210

[12] Weng X and Neethirajan S 2017 Trends Food Sci. Technol. 65 10

[13] Jokerst J C, Emory J M and Henry C S 2012 Analyst 13724

[14] Sia S K and Whitesides G M 2003 Electrophoresis 243563

[15] Ren K, Zhou J and Wu H 2013 Acc. Chem. Res. 462396

[16] Busa L S, Mohammadi S, Maeki M, Ishida A, Tani H and Tokeshi M 2016 Micromachines 786

[17] Dilna P, Raveendran J, Stanley J and Satheesh Babu T G 2018 Mater. Today Proc. 516220

[18] Carrilho E, Martinez A W and Whitesides G M 2009 Anal. Chem. 817091

[19] Dungchai W, Chailapakul O and Henry C S 2011 Analyst 136 77

[20] Mahayothee B, Udomkun P, Nagle M, Haewsungcharoen M, Janjai S and Mueller J 2009 Eur. Food Res. Technol. 229329

[21] Kuan C M, York R L and Cheng C M 2015 Sci. Rep. 518570

[22] Borin A, Ferrao M F, Mello C, Maretto D A and Poppi R J 2006 Anal. Chim. Acta 57925

[23] Dhar N R 1924 J. Phys. Chem. 28125 